Vietnam Journal of Mechanics, VAST, Vol.40, No. 1 (2018), pp. 15-32

DOI:10.15625/0866-7136/9341

\title{
STUDY OF THE INFLUENCE OF SMALL DEFECTS NEAR A SINGULAR POINT IN ANTIPLANE ELASTICITY BY AN ASYMPTOTIC METHOD
}

\author{
Dang Thi Bach Tuyet ${ }^{1}$, Laurence Halpern ${ }^{2}$, Jean-Jacques Marigo ${ }^{1, *}$ \\ ${ }^{1}$ Laboratoire de Mécanique des Solides, École Polytechnique, F-Palaiseau, France \\ ${ }^{2}$ LAGA, Université Paris XIII, F-Villetaneuse, France \\ *E-mail: marigo@lms.polytechnique.fr \\ Received March 16, 2017
}

\begin{abstract}
We consider a domain made of a linear elastic material which contains an angular point. A small defect, like a cavity or a crack, is located in the neighborhood of the tip of the wedge. In order to study its influence both on the local and global responses of the body, we use a matched asymptotic expansion method. After the general construction of the matched asymptotic expansions for an arbitrary defect, we develop the method in the particular case where the defect is a small crack. The numerical results obtained from the method are finally compared with those given by the classical finite element method. All the analysis is made in an antiplane setting in order to make easier the calculations.
\end{abstract}

Keywords: Brittle fracture, cohesive model, asymptotic methods, singularities.

\section{INTRODUCTION}

A major issue in fracture mechanics is how to model the initiation of a crack in a sound material, see [1-4]. If one uses Griffith criterion like in [5,6] or cohesive force models like in $[7,8]$, the main difficulty is to compute with a good accuracy mechanical quantities like the energy release rate associated with a crack of small length which appears at the tip of a notch, see [9-11]. The classical finite element method leads to inaccurate results because of the overlap of two singularities (one due to the tip of the notch, the other due to the tip of the crack) which cannot be correctly captured, see [12-14]. A specific method of approximation, like in [15-21], based on asymptotic expansions is preferable because the singularities are then obtained explicitly. The present paper is devoted to the presentation of this asymptotic method in the more general case of a defect (and not only of a crack) located at the tip of a notch in the simplified context of antiplane linear elasticity. It turns out that many works have been devoted to the study of elliptic problems in corner domains, see for example [22-24]. But none proposes a comprehensive method to compute with precision as large as it is needed the mechanical fields and all

(C) 2018 Vietnam Academy of Science and Technology 
related physical quantities in such a situation. Accordingly, we present here the method of construction of the matched asymptotic expansions first in the general case and then to the particular case of a noncohesive crack. The numerical results are finally compared with those given by the classical finite element method.

\section{THE REAL PROBLEM}

Here, we are interested to the case where a small geometrical defect of size $\ell$ (like a crack or a void) is located near the corner of a notch, see Fig. 1 . The geometry of the notch is characterized by its angle $\omega$, see Fig. 2. The angle of the notch is a parameter which can run in the interval $(0,2 \pi)$. The tip of the notch is taken as the origin of the space and we will consider two scales of coordinates: the "macroscopic" coordinates $\mathbf{x}=\left(x_{1}, x_{2}\right)$ which are used in the outer domain and the "microscopic" coordinates $\mathbf{y}=\mathbf{x} / \ell=\left(y_{1}, y_{2}\right)$ which are used in the neighborhood of the tip of the notch where the defect is located, see Fig. 2. The unit vector orthogonal to the $\left(x_{1}, x_{2}\right)$ plane is denoted $\mathbf{e}_{3}$.

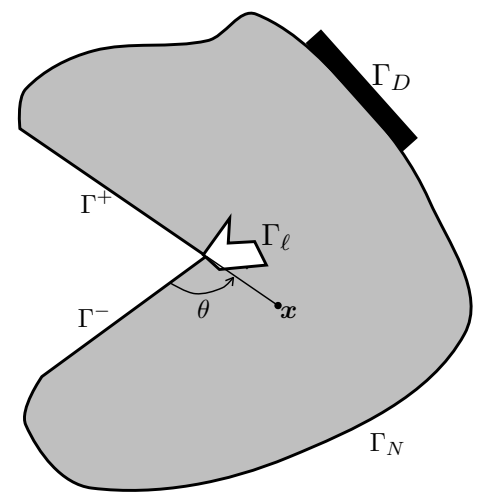

Fig. 1. The domain $\Omega_{\ell}$ for the real problem
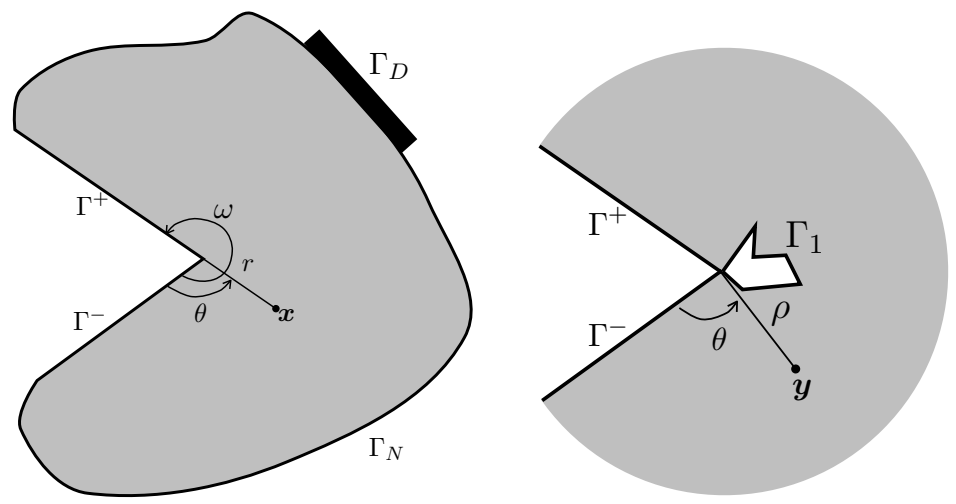

Fig. 2. The domains $\Omega_{0}$ (left) and $\Omega^{\infty}$ (right) for, respectively, the outer and the inner problems

The natural reference configuration of the sound two-dimensional body is denoted by $\Omega_{0}$ while the associated body which contains a defect of size $\ell$ is denoted by $\Omega_{\ell}$. Accordingly one has

$$
\Omega_{\ell}=\Omega_{0} \backslash \mathcal{D}_{\ell},
$$

where $\mathcal{D}_{\ell}$ denotes the domain occupied by the defect, the boundary of which is $\Gamma_{\ell}$. $\mathcal{D}_{\ell}$ is contained in the disk of center $(0,0)$ and radius $\ell$. (In the case of a crack, one has $\mathcal{D}_{\ell}=\Gamma_{\ell}$ ). The two edges of the notch are denoted by $\Gamma^{+}$and $\Gamma^{-}$, see Fig. 1 . When one uses polar coordinates $(r, \theta)$, the pole is the tip of the notch and the origin of the polar angle is the edge $\Gamma^{-}$. Accordingly, we have

$$
r=\|\mathbf{x}\|, \Gamma^{-}=\left\{(r, \theta), 0<r<r^{*}, \theta=0\right\}, \Gamma^{+}=\left\{(r, \theta), 0<r<r^{*}, \theta=\omega\right\} .
$$

This body is made of an elastic isotropic material whose shear modulus is $\mu>0$. It is submitted to a loading such that the displacement field at equilibrium $\mathbf{u}$ be antiplane, i.e.

$$
\mathbf{u}(\mathbf{x})=u^{\ell}\left(x_{1}, x_{2}\right) \mathbf{e}_{3},
$$


where the superscript $\ell$ is used in order to recall that the real displacement depends on the size of the defect. We assume that the body forces are zero and then $u^{\ell}$ must be a harmonic function in order to satisfy the equilibrium equations in the bulk

$$
\Delta u^{\ell}=0 \text { in } \Omega_{\ell},
$$

$\Delta$ representing the Laplacian differential operator.

The edges of the notch are free while $\Gamma_{\ell}$ is submitted to a density of (antiplane) surface forces. Accordingly, the boundary conditions on $\Gamma_{\ell}$ and $\Gamma^{ \pm}$read as

$$
\frac{\partial u^{\ell}}{\partial v}=0 \quad \text { on } \quad \Gamma^{ \pm}, \quad \frac{\partial u^{\ell}}{\partial v}=\lg (\mathbf{y}) \quad \text { on } \quad \Gamma_{\ell} .
$$

In (4), $v$ denotes the unit outer normal vector to the domain and we assume that the density of (antiplane) surface forces depends on the microscopic variable $\mathbf{y}$ and has a magnitude of the order of $\ell$.

The remaining part of the boundary of $\Omega_{\ell}$ is divided into two parts: $\Gamma_{D}$ where the displacement is prescribed and $\Gamma_{N}$ where (antiplane) surface forces are prescribed. Specifically, we have

$$
u^{\ell}=f(\mathbf{x}) \quad \text { on } \quad \Gamma_{D}, \quad \frac{\partial u^{\ell}}{\partial v}=h(\mathbf{x}) \quad \text { on } \quad \Gamma_{N} .
$$

The following Proposition is a characterization of functions which are harmonic in an angular sector and whose normal derivatives vanish on the edges of the sector. It is of constant use thereafter.

Proposition 1. Let $r_{1}$ and $r_{2}$ be such that $0 \leq r_{1}<r_{2} \leq+\infty$ and let $D_{r_{1}}^{r_{2}}$ be the angular sector

$$
\mathcal{D}_{r_{1}}^{r_{2}}=\left\{(r, \theta): r \in\left(r_{1}, r_{2}\right), \quad \theta \in(0, \omega)\right\} .
$$

Then any function $u$ which is harmonic in $D_{r_{1}}^{r_{2}}$ and which satisfies the Neumann condition $\partial u / \partial \theta=0$ on the sides $\theta=0$ and $\theta=\omega$ can be read as

$$
u(r, \theta)=a_{0} \ln (r)+d_{0}+\sum_{n \in \mathbb{N}_{*}}\left(a_{n} r^{-n \lambda}+d_{n} r^{n \lambda}\right) \cos (n \lambda \theta),
$$

with $\lambda=\pi / \omega$, whereas the $a_{n}$ 's and the $d_{n}$ 's constitute two sequences of real numbers which are characteristic of $u$.

Proof. Since the normal derivative vanishes at $\theta=0$ and $\theta=\omega, u(r, \theta)$ can be read as the following Fourier series $[25,26]$

$$
u(r, \theta)=\sum_{n \in \mathbb{N}} f_{n}(r) \cos (n \lambda \theta) .
$$

In order that $u$ be harmonic, the functions $f_{n}$ must satisfy $r^{2} f^{\prime \prime}{ }_{n}+r f^{\prime}{ }_{n}-n^{2} \lambda^{2} f_{n}=0$, for each $n$. One easily deduces that $f_{0}(r)=a_{0} \ln (r)+d_{0}$ and $f_{n}(r)=a_{n} r^{-n \lambda}+d_{n} r^{n \lambda}$ for $n \geq 1$. 


\section{THE MATCHING ASYMPTOTIC METHOD}

When the length $\ell$ of the defect is small by comparison with the characteristic length of the body (here, this characteristic length is equal to 1), then it is necessary to make an asymptotic analysis of the problem rather than to try to obtain directly an approximation by classical finite element methods. In the case of a crack for instance, because of the overlap of two singularities (one at the tip of the notch and the other at the tip of the crack), it is difficult and even impossible to obtain accurate results without using a relevant asymptotic method. Here we will use the matched asymptotic expansion technique which consists in making two asymptotic expansions of the field $u^{\ell}$ in terms of the small parameter $\ell$. The first one, called the inner expansion, is valid in the neighborhood of the tip of the notch, while the other, called the outer expansion, is valid far from this tip. These two expansions are matched in an intermediate zone.

\subsection{The outer expansion}

Far from the tip of the notch, i.e. for $r \gg \ell$, we assume that the real displacement field $u^{\ell}$ can be expanded as follows

$$
u^{\ell}(\mathbf{x})=\sum_{i \in \mathbb{N}} \ell^{i \lambda} u^{i}(\mathbf{x}) .
$$

In (7), even if this expansion is valid far enough from $r=0$ only, the fields $u^{i}$ must be defined in the whole outer domain $\Omega_{0}$ which corresponds to the sound body, see Fig. 2left. Inserting this expansion into the set of equations constituting the real problem, one obtains the following equations that the $u^{i}$ 's must satisfy:

The first outer problem $i=0$

$$
\left\{\begin{array}{lll}
\Delta u^{0}=0 & \text { in } & \Omega_{0} \\
\frac{\partial u^{0}}{\partial v}=0 & \text { on } & \Gamma^{+} \cup \Gamma^{-} \\
\frac{\partial u^{0}}{\partial v}=h(\mathbf{x}) & \text { on } & \Gamma_{N} \\
u^{0}=f(\mathbf{x}) & \text { on } & \Gamma_{D}
\end{array}\right.
$$

The other outer problems $i \geq 1$

Moreover, the behavior of $u^{i}$ in the neighborhood of $r=0$ is singular and the singularity will be given by the matching conditions.

\subsection{The inner expansion}

Near the tip of the notch, i.e. for $r \ll 1$, we assume that the real displacement field $u^{\ell}$ can be expanded as follows

$$
u^{\ell}(\mathbf{x})=\ln (\ell) \sum_{i \in \mathbb{N}} \ell^{i \lambda} w^{i}(\mathbf{y})+\sum_{i \in \mathbb{N}} \ell^{i \lambda} v^{i}(\mathbf{y}) .
$$

In (10), even if this expansion is valid only in the neighborhood of $r=0$, the fields $v^{i}$ and $w^{i}$ must be defined in the infinite inner domain $\Omega^{\infty}$. The domain $\Omega^{\infty}$ is the infinite angular sector $\mathcal{D}_{0}^{\infty}$ of the $\left(y_{1}, y_{2}\right)$ plane from which one removes the rescaled defect of size 1, see Fig. 2-right

$$
\Omega^{\infty}=\mathcal{D}_{0}^{\infty} \backslash \mathcal{D}_{1}
$$


The boundary of $\mathcal{D}_{1}$ is $\Gamma_{1}$. (In the case of a crack, one has $\mathcal{D}_{1}=\Gamma_{1}$.) Inserting this expansion into the set of equations constituting the real problem, one obtains the following equations that the $v^{i}$ 's must satisfy:

The first inner problem $i=0$

$$
\begin{cases}\Delta v^{0}=0 & \text { in } \Omega^{\infty} \\ \frac{\partial v^{0}}{\partial \theta}=0 & \text { on } \theta=0 \text { and } \theta=\omega \\ \frac{\partial v^{0}}{\partial v}=g(\mathbf{y}) & \text { on } \Gamma_{1}\end{cases}
$$

The other inner problems $i \geq 1$

$$
\left\{\begin{array}{l}
\Delta v^{i}=0 \text { in } \Omega^{\infty} \\
\frac{\partial v^{i}}{\partial \theta}=0 \text { on } \theta=0 \text { and } \theta=\omega \\
\frac{\partial v^{i}}{\partial v}=0 \text { on } \Gamma_{1}
\end{array}\right.
$$

The $w^{i \prime}$ s must satisfy, for every $i \geq 0$ the same equations as the $v^{i \prime s}$ for $i \geq 1$. To complete the set of equations one must add the behavior at infinity of the $v^{i}$ s and the $w^{i}$ s. This behavior will be given by the matching conditions with the outer problems.

\subsection{Matching conditions}

Since all the displacement fields $u^{i}$ are harmonic in the sector $\mathcal{D}_{0}^{r_{2}}$ and satisfy a homogeneous Neumann boundary condition on the edges of this angular sector, we can use Proposition 1. Accordingly, in $\mathcal{D}_{0}^{r_{2}}$ the field $u^{i}$ can read as

$$
u^{i}(\mathbf{x})=a_{0}^{i} \ln (r)+d_{0}^{i}+\sum_{n \in \mathbb{N}_{*}}\left(a_{n}^{i} r^{-n \lambda}+d_{n}^{i} r^{n \lambda}\right) \cos (n \lambda \theta) .
$$

In the same way for the inner expansion, since all the displacement fields $v^{i}$ and $w^{i}$ are harmonic in the sector $\mathcal{D}_{1}^{\infty}$ of the y plane and satisfy a homogeneous Neumann boundary condition on the edges of this angular sector, we can use Proposition 1 with the macroscopic coordinates $\mathbf{x}$ and $r$ replaced by the microscopic coordinates $\mathbf{y}$ and $\rho=$ $\|\mathbf{y}\|=r / \ell$. Accordingly, in $\mathcal{D}_{1}^{\infty}$ the fields $v^{i}$ and $w^{i}$ can read as

$$
\begin{aligned}
& v^{i}(\mathbf{y})=c_{0}^{i} \ln (\rho)+b_{0}^{i}+\sum_{n \in \mathbb{N}_{*}}\left(c_{n}^{i} \rho^{-n \lambda}+b_{n}^{i} \rho^{n \lambda}\right) \cos (n \lambda \theta), \\
& w^{i}(\mathbf{y})=e_{0}^{i} \ln (\rho)+f_{0}^{i}+\sum_{n \in \mathbb{N}_{*}}\left(e_{n}^{i} \rho^{-n \lambda}+f_{n}^{i} \rho^{n \lambda}\right) \cos (n \lambda \theta) .
\end{aligned}
$$

The outer expansion and the inner expansion are both valid in any intermediate zone $\mathcal{D}_{r_{1}}^{r_{2}}$ such that $\ell \ll r_{1}<r_{2} \ll 1$. Inserting (14) into the outer expansion (7) with $r=\ell \rho$ leads to

$$
u^{\ell}(\mathbf{x})=\sum_{i \in \mathbb{N}} \ln (\ell) \ell^{i \lambda} a_{0}^{i}+\sum_{i \in \mathbb{N}} \ell^{i \lambda}\left(a_{0}^{i} \ln (\rho)+d_{0}^{i}+\sum_{n \in \mathbb{N}_{*}}\left(a_{n}^{i+n} \rho^{-n \lambda}+d_{n}^{i-n} \rho^{n \lambda}\right) \cos (n \lambda \theta)\right),
$$


with the convention that $d_{n}^{i-n}=0$ when $n>i$. Inserting (15) and (16) into the inner expansion (10) leads to

$$
\begin{aligned}
u^{\ell}(\mathbf{x})= & \sum_{i \in \mathbb{N}} \ln (\ell) \ell^{i \lambda}\left(e_{0}^{i} \ln (\rho)+f_{0}^{i}+\sum_{n \in \mathbb{N}_{*}}\left(e_{n}^{i} \rho^{-n \lambda}+f_{n}^{i} \rho^{n \lambda}\right) \cos (n \lambda \theta)\right) \\
& +\sum_{i \in \mathbb{N}} \ell^{i \lambda}\left(c_{0}^{i} \ln (\rho)+b_{0}^{i}+\sum_{n \in \mathbb{N}_{*}}\left(c_{n}^{i} \rho^{-n \lambda}+b_{n}^{i} \rho^{n \lambda}\right) \cos (n \lambda \theta)\right) .
\end{aligned}
$$

Both expansions (17) and (18) are valid provided that $1 \ll \rho \ll 1 / \ell$. By identification one gets the following properties for the coefficients of the inner and outer expansions, see Tab. 1.

Table 1. The relations between the coefficients of the inner and outer expansions given by the matching conditions

\begin{tabular}{ll}
\hline$e_{n}^{i}=0$ & $i \geq 0, n \geq 0$ \\
\hline$f_{0}^{i}=a_{0}^{i}$ & $i \geq 0$ \\
\hline$f_{n}^{i}=0$ & $i \geq 0, n \geq 0$ \\
\hline$a_{n}^{i}=0$ & $n>i \geq 0$ \\
\hline$c_{n}^{i}=a_{n}^{i+n}$ & $i \geq 0, n \geq 0$ \\
\hline$b_{n}^{i}=0$ & $n>i \geq 0$ \\
\hline$d_{n}^{i}=b_{n}^{i+n}$ & $i \geq 0, n \geq 0$ \\
\hline
\end{tabular}

Remark 1. One deduces from Tab. 1 that the fields $w^{i}$ are constant in the whole inner domain:

$$
w^{i}(\mathbf{y})=a_{0}^{i}, \quad \forall \mathbf{y} \in \Omega^{\infty}, \quad \forall i \geq 0 .
$$

Therefore, these fields will be determined once the constants $a_{0}^{i}$ will be known.

\subsection{The singular behavior of the $u^{i}$ s and the $v^{i \text { 's }}$}

We deduce from the matching conditions the behavior of $u^{i}$ in the neighborhood of $r=0$ and the behavior of $v^{i}$ in the neighborhood of $\rho=\infty$. In particular, one obtains the form of their singularities. Let us first precise what one means by singularity.

Definition 1. A field $u$ defined in $\Omega_{0}$ is said regular in $\Omega_{0}$ if $u \in H^{1}\left(\Omega_{0}\right)$, i.e. $u \in L^{2}\left(\Omega_{0}\right)$ and $\nabla u \in L^{2}\left(\Omega_{0}\right)^{2}$ [25]. It is said singular otherwise.

A field $u$ defined in the unbounded sector $\Omega^{\infty}$ is said regular in $\Omega^{\infty}$ if $\nabla u \in L^{2}\left(\Omega^{\infty}\right)^{2}$ and $\lim _{\rho \rightarrow \infty} u(\rho, \theta)=0$. It is said singular otherwise.

By virtue of the analysis of the previous subsection, the field $u^{0}$ can be read in a neighborhood of the tip of the notch as

$$
u^{0}(\mathbf{x})=a_{0}^{0} \ln (r)+\sum_{n \in \mathbb{N}} b_{n}^{n} r^{n \lambda} \cos (n \lambda \theta) .
$$


Since $\ln (r)$ is singular in $\Omega_{0}$ whereas $r^{n \lambda} \cos (n \lambda \theta)$ is regular (for $n \geq 0$ ) in $\Omega_{0}$ in the sense of Definition 1, $a_{0}^{0} \ln (r)$ can be considered as the singular part of the field $u^{0}$. Accordingly, one can decompose $u^{0}$ into its singular and its regular part as follows

$$
\begin{gathered}
u^{0}(\mathbf{x})=u_{S}^{0}(\mathbf{x})+\bar{u}^{0}(\mathbf{x}), \\
u_{S}^{0}(\mathbf{x})=a_{0}^{0} \ln (r), \quad \bar{u}^{0}(\mathbf{x}) \in H^{1}\left(\Omega_{0}\right) .
\end{gathered}
$$

In the same way, for $i \geq 1$, the field $u^{i}$ can be read in a neighborhood of the tip of the notch as

$$
u^{i}(\mathbf{x})=a_{0}^{i} \ln (r)+\sum_{n=1}^{i} a_{n}^{i} r^{-n \lambda} \cos (n \lambda \theta)+\sum_{n \in \mathbb{N}} b_{n}^{i+n} r^{n \lambda} \cos (n \lambda \theta) .
$$

Since $r^{-n \lambda} \cos (n \lambda \theta)$ is singular (for $n \geq 0$ ) in the sense of Definition 1, one can decompose $u^{i}$ into its singular and its regular part as follows

$$
\begin{gathered}
u^{i}(\mathbf{x})=u_{S}^{i}(\mathbf{x})+\bar{u}^{i}(\mathbf{x}), \\
u_{S}^{i}(\mathbf{x})=a_{0}^{i} \ln (r)+\sum_{n=1}^{i} a_{n}^{i} r^{-n \lambda} \cos (n \lambda \theta), \quad \bar{u}^{i} \in H^{1}\left(\Omega_{0}\right) .
\end{gathered}
$$

For the fields $v^{i}$ of the inner expansion, one has to study their behavior at infinity. By virtue of the analysis of the previous subsection, the field $v^{i}$ for $i \geq 0$ can be read in a neighborhood of $\rho=\infty$ as

$$
v^{i}(\mathbf{y})=a_{0}^{i} \ln (\rho)+\sum_{n=0}^{i} b_{n}^{i} \rho^{n \lambda} \cos (n \lambda \theta)+\sum_{n \in \mathbb{N}_{*}} a_{n}^{i+n} \rho^{-n \lambda} \cos (n \lambda \theta) .
$$

The field $\ln (\rho)$ as well as the fields $\rho^{n \lambda} \cos (n \lambda \theta)$, for $n \geq 0$, are singular in $\Omega^{\infty}$ in the sense of Definition 1 (even the constant field 1 corresponding to $n=0$ is singular). Since the fields $\rho^{-n \lambda} \cos (n \lambda \theta)$ are regular when $n \geq 1, a_{0}^{i} \ln (\rho)+\sum_{n=0}^{i} b_{n}^{i} \rho^{n \lambda} \cos (n \lambda \theta)$ can be considered as the singular part of the field $v^{i}$. Accordingly, one can decompose $v^{i}$ into its singular and its regular part as follows

$$
\begin{gathered}
v^{i}(\mathbf{y})=v_{S}^{i}(\mathbf{y})+\bar{v}^{i}(\mathbf{y}), \\
v_{S}^{i}(\mathbf{y})=a_{0}^{i} \ln (\rho)+\sum_{n=0}^{i} b_{n}^{i} \rho^{n \lambda} \cos (n \lambda \theta), \quad \nabla \bar{v}^{i} \in L^{2}\left(\Omega^{\infty}\right), \quad \lim _{|\mathbf{y}| \rightarrow \infty} \bar{v}^{i}(\mathbf{y})=0 .
\end{gathered}
$$

Remark 2. This analysis of the singularities shows that the singular parts of the fields $u^{i}$ and $v^{i}$ will be known once the coefficients $a_{n}^{i}$ and $b_{n}^{i}$ will be determined for $0 \leq n \leq i$. 


\subsection{The problems giving the regular parts $\bar{u}^{i}$ and $\bar{v}^{i}$}

We are now in position to set the inner and outer problems giving the fields $v^{i}$ and $u^{i}$. Since, by construction, the singular parts of these fields are harmonic and satisfy the homogeneous Neumann boundary conditions on the edges of the notch, their regular parts must verify the following boundary value problems.

The first outer problem, $i=0$

Find $\bar{u}^{0}$ regular in $\Omega^{0}$ such that

$$
\begin{cases}\Delta \bar{u}^{0}=0 & \text { in } \Omega_{0} \\ \frac{\partial \bar{u}^{0}}{\partial v}=0 & \text { on } \Gamma^{+} \cup \Gamma^{-} \\ \frac{\partial \bar{u}^{0}}{\partial v}=h-\frac{\partial u_{S}^{0}}{\partial v} & \text { on } \Gamma_{N} \\ \bar{u}^{0}=f-u_{S}^{0} & \text { on } \Gamma_{D}\end{cases}
$$

The first inner problem, $i=0$

Find $\bar{v}^{0}$ regular in $\Omega^{\infty}$ such that

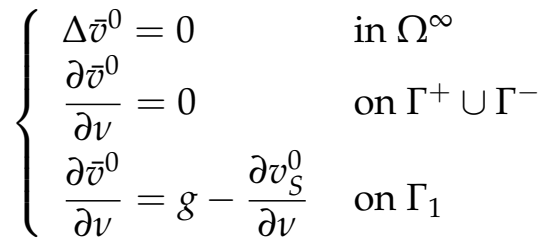

The other outer problems, $i \geq 1$

Find $\bar{u}^{i}$ regular in $\Omega^{0}$ such that

Let us study first the outer problems. We have the following proposition which is a direct consequence of classical results for the Laplace equation [25]:

Proposition 2. Let $i \geq 0$. For a given singular part $u_{S}^{i}$, i.e. if the coefficients $a_{n}^{i}$ are known for all $n$ such that $0 \leq n \leq i$, then there exists a unique solution $\bar{u}^{i}$ of (30) (or of (29) when $i=0$ ). Consequently, the coefficients $b_{n}^{i+n}$ are then determined for all $n \geq 0$.

Let us consider now the inner problems. We obtain the following proposition

Proposition 3. Let $i \geq 0$. For given $b_{n}^{i}$ with $0 \leq n \leq i$, there exists a regular solution $v^{i}$ for the $i$-th inner problem if and only is the coefficient $a_{0}^{i}$ is such that

$$
a_{0}^{0}=-\frac{1}{\omega} \int_{\Gamma_{1}} g(s) d s, \quad a_{0}^{i}=0 \quad \text { for } \quad i \geq 1 .
$$

Moreover, if this condition is satisfied, then the solution is unique and therefore the coefficients $a_{n}^{i+n}$ are determined for all $n \geq 0$.

Proof. The inner problems are pure Neumann problems in which no Dirichlet boundary conditions are imposed to the $v^{i}$ s. Consequently, they admit a solution (if and) only if the Neumann data satisfy a global compatibility condition. Let us re-establish that condition. Let $\Omega^{R}$ be the part of $\Omega^{\infty}$ included in the ball of radius $R>1$, i.e. $\Omega^{R}=$ $\Omega^{\infty} \cap\{\mathbf{y}:|\mathbf{y}|<R\}$. Let us consider first the case $i=0$. Integrating the equation $\Delta v^{0}=0$ 
over $\Omega^{R}$ and using the boundary conditions leads to

$$
0=\int_{\partial \Omega^{R}} \frac{\partial v^{0}}{\partial v} d s=\int_{0}^{\omega} \frac{\partial v^{0}}{\partial \rho}(R, \theta) R d \theta+\int_{\Gamma_{1}} g(s) d s .
$$

Using (26), one gets $R \frac{\partial v^{0}}{\partial \rho}(R, \theta)=a_{0}^{0}+\sum_{n \in \mathbb{N}_{*}} n \lambda\left(-c_{n}^{0} R^{-n \lambda}+b_{n}^{0} R^{n \lambda}\right) \cos (n \lambda \theta)$. Since $\int_{0}^{\omega} \cos (n \lambda \theta) d \theta=0$ for all $n \geq 1$, after inserting in (34) one obtains the desired condition for $a_{0}^{0}$. One proceeds exactly in the same manner for $i \geq 1$ and one obtains the desired condition because the integral over $\Gamma_{1}$ vanishes.

If the compatibility condition (33) is satisfied, then one proves the existence of a regular solution for $\bar{v}^{i}$ by standard arguments, [25]. Note however that, since $\nabla \bar{v}^{i}$ belongs to $L^{2}\left(\Omega^{\infty}\right), \bar{v}^{i}$ tends to a constant at infinity and this constant is fixed to 0 by the additional regularity condition. As far as the uniqueness is concerned, the solution of this pure Neumann problem is unique up to a constant and the constant is fixed by the condition that $\bar{v}^{i}$ vanishes at infinity.

Once $v^{i}$ is determined, one obtains the coefficients $a_{n}^{i+n}$ by virtue of Proposition 1 and (26).

Remark 3. If the forces applied to the boundary of the defect are equilibrated, i.e. if $\int_{\Gamma_{1}} g(s) d s=$ 0 , then all the coefficients $a_{0}^{i}$ vanish and hence the terms in $\ln (\ell)$ disappear in the inner expansion. There is no more logarithmic singularities in the $u^{i}$ 's and the $v^{i}$ 's.

\subsection{The construction of the outer and inner expansions}

Equipped with the previous results, we are in position to explain how one can determine the different terms of the two expansions. Let us explain first how one obtains the first terms.

(1) One obtains $a_{0}^{0}$ by (33) and hence one knows $u_{S}^{0}$.

(2) Knowing $u_{S}^{0}$, one determines $\bar{u}^{0}$ and hence $u^{0}$ by solving (29), see Proposition 2.

(3) Knowing $u^{0}$, one calculates $b_{n}^{n}$ for $n \geq 0$ as a regular part of $u^{0}$, see the next subsection for the practical method. Hence, one knows $v_{S}^{0}$.

(4) Knowing $v_{S}^{0}$, one determines $\bar{v}^{0}$ and hence $v^{0}$ by solving (31), see Proposition 3.

(5) Knowing $v^{0}$, one calculates $a_{n}^{n}$ for $n \geq 1$ as a regular part of $v^{0}$, see the next subsection for the practical method. Hence, since $a_{0}^{1}=0$, one knows $u_{S}^{1}$.

Then one proceeds by induction. Let $i \geq 1$. Assuming that the following properties hold true,

H1 $u^{j}$ and $v^{j}$ have been determined for $0 \leq j \leq i-1$

$\mathrm{H} 2 b_{n}^{j}$ is known for $0 \leq n \leq j \leq i-1$

H3 $a_{n}^{j+n}$ and $b_{n}^{j+n}$ are known for $0 \leq j \leq i-1$ and $n \geq 0$

H4 $a_{n}^{j}$ is known for $0 \leq n \leq j \leq i$

let us prove that they remain true for $i+1$.

C1 Knowing $a_{n}^{i}$ for $0 \leq n \leq i$, one knows $u_{S}^{i}$. Knowing $u_{S}^{i}$, one determines $\bar{u}^{i}$ and hence $u^{i}$ by solving (30), see Proposition 2 . 
C2 Knowing $u^{i}$, one calculates $b_{n}^{j+n}$ for $n \geq 0$ as a regular part of $u^{i}$, see the next subsection for the practical method. Hence, one knows $v_{S}^{i}$.

C3 Since $b_{0}^{i}$ is known and since $b_{n}^{i}=b_{n}^{j+n}$ with $j=i-n$, one knows $b_{n}^{i}$ for $0 \leq n \leq i$.

C4 Knowing $v_{S}^{i}$, one determines $\bar{v}^{i}$ and hence $v^{i}$ by solving (32), see Proposition 3.

C5 One knows that $a_{0}^{i}=0$. Knowing $v^{i}$, one calculates $a_{n}^{i+n}$ for $n \geq 1$ as a regular part of $v^{i}$, see the next subsection for the practical method.

C6 Since $a_{0}^{i+1}=0$ and since $a_{n}^{i+1}=a_{n}^{j+n}$ with $j=i+1-n$, one knows $a_{n}^{i+1}$ for $0 \leq n \leq i+1$.

This iterative method is summarized in Tab. 2.

Table 2. Summary of the inductive method to obtain the coefficients $a_{n}^{i}$ and $b_{n}^{i}$ : in each cell is indicated the problem which must be solved

\begin{tabular}{|c|c|c|c|c|c|}
\hline$a_{n}^{i} / b_{n}^{i}$ & $i=0$ & $i=1$ & $i=2$ & $i=3$ & $i=4$ \\
\hline$n=0$ & (33)/Outer 0 & 0/Outer 1 & 0/Outer 2 & $0 /$ Outer 3 & $0 /$ Outer 4 \\
\hline$n=1$ & 0 & Inner $0 /$ Outer 0 & Inner $1 /$ Outer 1 & Inner $2 /$ Outer 2 & Inner $3 /$ Outer 3 \\
\hline$n=2$ & 0 & 0 & Inner $0 /$ Outer 0 & Inner $1 /$ Outer 1 & Inner $2 /$ Outer 2 \\
\hline$n=3$ & 0 & 0 & 0 & Inner $0 /$ Outer 0 & Inner 1 /Outer 1 \\
\hline$n=4$ & 0 & 0 & 0 & 0 & Inner $0 /$ Outer 0 \\
\hline
\end{tabular}

3.7. The practical method for determining the coefficients $a_{n}^{i}$ and $b_{n}^{i}$ for $0 \leq n \leq i$

Throughout this section, $\mathcal{C}_{r}$ denotes the arc of circle of radius $r$ starting on $\Gamma^{-}$and ending on $\Gamma^{+}$

$$
\mathcal{C}_{r}=\{(r, \theta): 0 \leq \theta \leq \omega\} .
$$

The coefficients $a_{n}^{i}$ and $b_{n}^{i}$ can be obtained by path integrals (which are path independent like in [27]) as it is proved in the following proposition.

Proposition 4. Let $i \geq 0$ and let us assume that the $i^{\text {ths }}$ inner and outer problems are solved and thus that $\bar{v}^{i}$ and $\bar{u}^{i}$ are known. Then

(1) For $n \geq 1, a_{n}^{i+n}$ is given by the following path integral over $\mathcal{C}_{\rho}$ which is independent of $\rho$ provided that $\rho>1$

$$
a_{n}^{i+n}=\frac{2 \rho^{n \lambda}}{\omega} \int_{0}^{\omega} \bar{v}^{i}(\rho, \theta) \cos (n \lambda \theta) d \theta .
$$

(2) For $n \geq 0, b_{n}^{i+n}$ is given by the following path integral over $\mathcal{C}_{r}$ which is independent of $r$ provided that $0<r<r^{*}$

$$
b_{0}^{i}=\frac{1}{\omega} \int_{0}^{\omega} \bar{u}^{i}(r, \theta) d \theta, \quad b_{n}^{i+n}=\frac{2 r^{-n \lambda}}{\omega} \int_{0}^{\omega} \bar{u}^{i}(r, \theta) \cos (n \lambda \theta) d \theta, \quad \text { for } \quad n \geq 1 .
$$

Proof. The proofs are identical for the two families of coefficients and then one gives only the proof for $b_{n}^{i+n}$. By virtue of (23), the regular part $\bar{u}^{i}$ of $u^{i}$ is given by

$$
\bar{u}^{i}(r, \theta)=\sum_{p \in \mathbb{N}} b_{p}^{i+p} r^{p \lambda} \cos (p \lambda \theta) d \theta,
$$


for $0<r<r^{*}$. Since $\int_{0}^{\omega} \cos (p \lambda \theta) d \theta$ is equal to $\omega$ if $p=0$ and is equal to 0 otherwise, one obtains the expression for $b_{0}^{i}$. For $n \geq 1$, since $\int_{0}^{\omega} \cos (p \lambda \theta) \cos (n \lambda \theta) d \theta$ is equal to $\omega / 2$ if $p=n$ and is equal to 0 otherwise, one obtains the expression for $b_{n}^{i+n}$.

\section{VERIFICATION IN THE CASE OF A CIRCULAR CAVITY}

This section is devoted to an illustration of the asymptotic method in the case where the exact solution is known. That will allow us to prove the pertinency and the accuracy of the method. Specifically, we consider a Laplace problem posed in a domain which has the shape of an angular sector of angle $\omega \in(0,2 \pi)$ limited by two arcs of circle, see Fig. 3. The radius of the outer circle $\Gamma_{D}$ is equal to 1 whereas the radius of the inner circle $\Gamma_{\ell}$ is equal to $\ell$. Accordingly, the defect is a small circular cavity of radius $\ell$ centered at the origin. The notch edges $\Gamma_{\ell}^{-}=\left\{\mathbf{x}=r \mathbf{e}_{1}, r \in(\ell, 1)\right\}, \Gamma_{\ell}^{+}=$ $\left\{\mathbf{x}=r \cos \omega \mathbf{e}_{1}+r \sin \omega \mathbf{e}_{2}, r \in(\ell, 1)\right\}$ and the inner circle $\Gamma_{\ell}=\left\{\mathbf{x}=\ell \cos \theta \mathbf{e}_{1}+\ell \sin \theta \mathbf{e}_{2}\right.$, $\theta \in(\ell, \omega)\}$ are free, what leads to homogeneous Neumann boundary conditions

$$
\frac{\partial u^{\ell}}{\partial v}=0 \quad \text { on } \quad \Gamma_{\ell}^{+} \cup \Gamma_{\ell}^{-} \cup \Gamma_{\ell} .
$$

On the outer circle $\Gamma_{D}=\left\{\mathbf{x}=\cos \theta \mathbf{e}_{1}+\sin \theta \mathbf{e}_{2}, \theta \in(0, \omega)\right\}$ is applied a Dirichlet boundary condition

$$
u^{\ell}(\mathbf{x})=\cos \lambda \theta \quad \text { on } \quad \Gamma_{D}, \lambda=\frac{\pi}{\omega} .
$$

Let us note that this Dirichlet boundary condition on $\Gamma_{D}$ is compatible with the Neumann boundary condition on $\Gamma_{\ell}^{ \pm}$. Accordingly, the solution is obtained in a closed form which can read as

$$
u^{\ell}(\mathbf{x})=\left(\frac{\ell^{2 \lambda}}{1+\ell^{2 \lambda}} r^{-\lambda}+\frac{1}{1+\ell^{2 \lambda}} r^{\lambda}\right) \cos \lambda \theta
$$

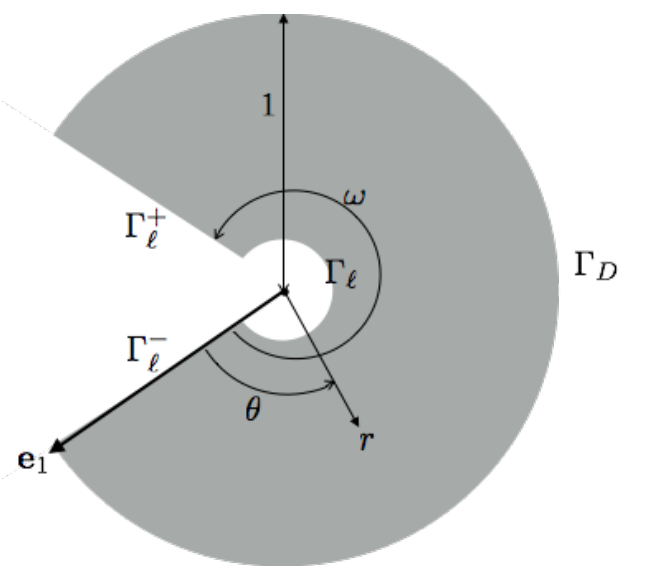

Fig. 3. The domain $\Omega_{\ell}$ in the case of a circular cavity 
Therefore, assuming that $\ell$ is small, the solution can be expanded for a given $r>0$ as follows

$$
u^{\ell}(\mathbf{x})=r^{\lambda} \cos \lambda \theta+\left(\sum_{i=1}^{\infty} \ell^{2 i \lambda}\right)\left(r^{-\lambda}-r^{\lambda}\right) \cos \lambda \theta .
$$

Introducing the rescaled coordinates $\mathbf{y}=\mathbf{x} / \ell$, the polar coordinate $r$ becomes $\rho:=$ $\|\mathbf{y}\|=r / \ell$ whereas $\theta$ remains unchanged. Inserting into (39), we get

$$
u^{\ell}(\mathbf{x})=\frac{\ell^{\lambda}}{1+\ell^{2 \lambda}}\left(\rho^{-\lambda}+\rho^{\lambda}\right) \cos \lambda \theta .
$$

Consequently, for a given $\rho>1$, the solution can be expanded as follows

$$
u^{\ell}(\mathbf{x})=\left(\sum_{i=1}^{\infty}(-1)^{i-1} \ell^{(2 i-1) \lambda}\right)\left(\rho^{-\lambda}+\rho^{\lambda}\right) \cos (\lambda \theta) .
$$

In other words, direct calculations from the known exact solution lead to the inner and outer expansions shown in Tab. 3.

Table 3. The outer expansions and inner expansions in the case of a circular cavity

\begin{tabular}{lll}
\hline rank & Outer expansion & Inner expansion \\
\hline$i=0$ & $u^{0}(\mathbf{x})=r^{\lambda} \cos \lambda \theta$ & $v^{0}(\mathbf{y})=0$ \\
$i \geq 1$ & $u^{2 i-1}(\mathbf{x})=0$ & $v^{2 i-1}(\mathbf{y})=(-1)^{i-1}\left(\rho^{-\lambda}+\rho^{\lambda}\right) \cos \lambda \theta$ \\
$i \geq 1$ & $u^{2 i}(\mathbf{x})=\left(r^{-\lambda}-r^{\lambda}\right) \cos \lambda \theta$ & $v^{2 i}(\mathbf{y})=0$ \\
\hline
\end{tabular}

Let us check that we recover the same expansions by following the procedure described in Section 3. We are in the situation where $\Gamma_{N}$ is empty, $g=0, f=\cos \lambda \theta$ and

$$
\Omega_{0}=\left\{\mathbf{x}=r \cos \theta e_{1}+r \sin \theta e_{2}, 0<r<1,0<\theta<\omega\right\} .
$$

(1) Since $g=0$, (33) gives $a_{0}^{0}=0$ and hence $u_{S}^{0}=0$.

(2) Solving (29) gives $\bar{u}^{0}=r^{\lambda} \cos \lambda \theta$.

(3) Using (36) gives $b_{1}^{1}=1$ and $b_{n}^{n}=0$ for $n \neq 1$. Since $b_{0}^{0}=0$, one gets $v_{S}^{0}=0$.

(4) Solving (31) gives $\bar{v}^{0}=0$.

(5) Using (35) gives $a_{n}^{n}=0$ for any $n$. Since $a_{1}^{1}=0$, one gets $u_{S}^{1}=0$.

(6) Solving (30) for $i=1$ gives $\bar{u}^{1}=0$.

(7) Using (36) gives $b_{n}^{n+1}=0$ for any $n$. Since $b_{1}^{1}=1$, one gets $v_{S}^{1}=\rho^{\lambda} \cos \lambda \theta$.

(8) Solving (32) for $i=1$ gives $\bar{v}^{1}=\rho^{-\lambda} \cos \lambda \theta$.

(9) Using (35) gives $a_{1}^{2}=1$ and $a_{n}^{n+1}=0$ for $n \neq 1$. Therefore $u_{S}^{2}=r^{-\lambda} \cos \lambda \theta$.

(10) Solving (30) for $i=2$ gives $\bar{u}^{2}=-r^{\lambda} \cos \lambda \theta$.

(11) Using (36) gives $b_{1}^{3}=-1$ and $b_{n}^{n+2}=0$ for $n \neq 1$. Therefore $v_{S}^{2}=0$.

(12) Solving (32) for $i=2$ gives $\bar{v}^{2}=0$.

Then, by induction, we recover the same expansions as those shown in Tab. 3 . The end of the verification is left to the reader. 


\section{CASE OF A CRACK}

In this section, the method is applied to the case where the defect is a (non cohesive) crack. Specifically, let $\Omega$ be the rectangle $(-H, L) \times(-H,+H)$. We remove from $\Omega$ the following sector $\mathcal{N}_{\epsilon}$ where $0<\epsilon<1$

$$
\mathcal{N}_{\epsilon}=\left\{\mathbf{x}=\left(x_{1}, x_{2}:-H<x_{1} \leq 0,\left|x_{2}\right| \leq \epsilon\left|x_{1}\right|\right)\right\} .
$$

So we obtain the notch-shaped body $\Omega_{0}=\Omega \backslash \mathcal{N}_{\epsilon}$. (Note that the limit case $\epsilon=0$ would correspond to a body with a preexisting crack of length $H$. We exclude a such case here so that the exponent of the singularity at the tip of the wedge be $\lambda=\pi / \omega>1 / 2$.) Finally we remove from $\Omega_{0}$ the segment line $\Gamma_{\ell}=(0, \ell) \times\{0\}$ and obtains the cracked body $\Omega_{\ell}$, see Fig. 4 . (Note that here the axes are chosen so that the crack be on the $x_{1}$-axis. We do not use polar coordinates.)

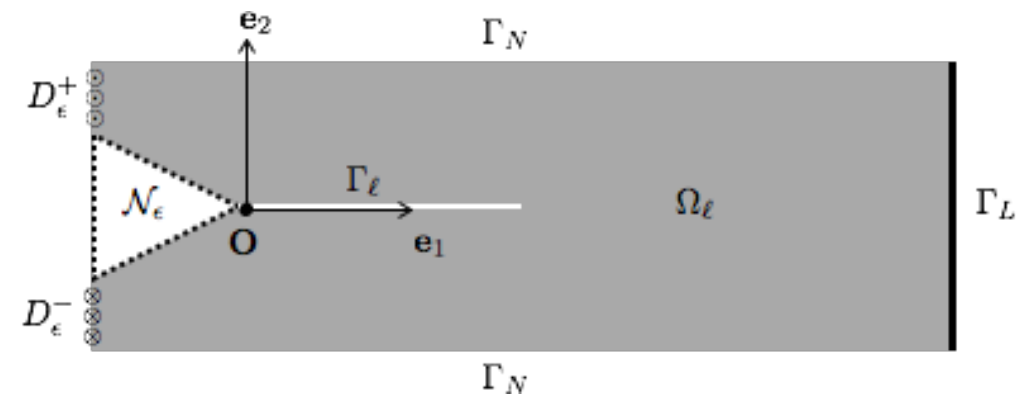

Fig. 4. The cracked notch-shaped body $\Omega_{\ell}$

The boundary $\Gamma_{D}$ where the displacement is prescribed corresponds to the sides $D_{\epsilon}^{ \pm}$ and $\Gamma_{L}$. Specifically, the boundary conditions read as

$$
u^{\ell}(\mathbf{x})= \begin{cases}+1 & \text { on } D_{\epsilon}^{+}=\{-H\} \times\{\epsilon H, H\} \\ -1 & \text { on } D_{\epsilon}^{-}=\{-H\} \times\{-H,-\epsilon H\} \\ 0 & \text { on } \Gamma_{L}=\{L\} \times\{-H, H\}\end{cases}
$$

whereas the remaining parts of the boundary (including the lips of the crack) are free. (In the case of a cohesive crack, the lips of the crack would be not free but submitted to cohesive forces depending in general of the jump of the displacement. Here we ignore such cohesive forces.) Accordingly, we have

$$
\frac{\partial u^{\ell}}{\partial x_{2}}=0 \quad \text { on } \quad \Gamma_{\ell} .
$$

We are in the case where $g=0$ on $\Gamma_{\ell}$. Therefore, by virtue of Proposition 3 , all the coefficients $a_{0}^{i}$ vanish and there is no logarithmic singularities. Accordingly, the solution can be expanded as follows

$$
\begin{array}{ll}
\text { Outer expansion } & u^{\ell}(\mathbf{x})=u^{0}(\mathbf{x})+\ell^{\lambda} u^{1}(\mathbf{x})+\ell^{2 \lambda} u^{2}(\mathbf{x})+\ell^{3 \lambda} u^{3}(\mathbf{x})+\ldots \\
\text { Inner expansion } & u^{\ell}(\mathbf{y})=v^{0}(\mathbf{y})+\ell^{\lambda} v^{1}(\mathbf{y})+\ell^{2 \lambda} v^{2}(\mathbf{y})+\ell^{3 \lambda} v^{3}(\mathbf{y})+\ldots
\end{array}
$$


with

$$
\lambda=\frac{\pi}{\omega}, \quad \omega=2 \pi-\arctan (2 \epsilon) .
$$

Remark 4. By symmetry of the geometry and the loading, the real field $u^{\ell}$ is an odd function of $x_{2}$, i.e.

$$
u^{\ell}\left(x_{1},-x_{2}\right)=-u^{\ell}\left(x_{1}, x_{2}\right) .
$$

Therefore, all the fields $u^{i}, \bar{u}^{i}, v^{i}, \bar{v}^{i}$ admit the same symmetry. One deduces from Proposition 4 that all the coefficients $b_{2 n}^{i+2 n}$ and $a_{2 n}^{i+2 n}$ vanish. We can also prove (but the proof is too long to be reproduced here) that all the odd terms of the outer expansion and all the even terms of the inner expansions vanish, i.e. $u^{2 i+1}=0$ and $v^{2 i}=0$ for all $i \in \mathbb{N}$.

The main goal of this section is to obtain an accurate value of the elastic energy stored in the cracked body, say $\mathcal{P}^{\ell}$, for small values of $\ell$ by using the matched asymptotic method because it is difficult to evaluate the accuracy of its values obtained by a classical finite element method. By definition, the elastic energy is given by

$$
\mathcal{P}^{\ell}=\frac{1}{2} \int_{\Omega_{\ell}} \mu \nabla u^{\ell} \cdot \nabla u^{\ell} d x .
$$

By virtue of Clapeyron's formula, the elastic energy at equilibrium is equal to one half of the work done by the external loads which are involved on $D_{\epsilon}^{ \pm}$to prescribe the displacement. Therefore, using the symmetry of $u^{\ell}$, the elastic energy can read as the following integral over $D_{\epsilon}^{+}$

$$
\mathcal{P}^{\ell}=-\int_{\epsilon H}^{H} \mu \frac{\partial u^{\ell}}{\partial x_{1}}\left(-H, x_{2}\right) d x_{2}
$$

which involves only the displacement field far from the tip of the notch. Accordingly, one can expand $\mathcal{P}^{\ell}$ by using the outer expansion of $u^{\ell}$. That leads to

$$
\mathcal{P}^{\ell}=\sum_{i \in \mathbb{N}} P^{2 i} \ell^{2 i \lambda} \quad \text { with } \quad P^{2 i}=-\int_{\epsilon H}^{H} \mu \frac{\partial u^{2 i}}{\partial x_{1}}\left(-H, x_{2}\right) d x_{2} .
$$

An important quantity for the study of the propagation of the crack is the energy release rate $\mathcal{G}^{\ell}$ which is the opposite of the derivative of the elastic energy with respect to the length of the crack, see $[5,9-11,28-30]$

$$
\mathcal{G}^{\ell}=\frac{d \mathcal{P}^{\ell}}{d \ell} .
$$

Its expansion can be immediately deduce from that of the energy

$$
\mathcal{G}^{\ell}=-\sum_{i \in \mathbb{N}_{*}} 2 i \lambda P^{2 i} \ell^{2 i \lambda-1} .
$$

To obtain the $i^{\text {th }}$ term of the expansion of $\mathcal{P}^{\ell}$ and $\mathcal{G}^{\ell}$, one must determine both the singular part $u_{S}^{i}$ and the regular part $\bar{u}^{i}$ of $u^{i}$. The singular part involves the coefficients $a_{n}^{i}$ for $1 \leq n \leq i$ which are obtained as the regular parts of the $v^{j}$ 's for $j \leq i$, see Subsection 3.6. Therefore, one must also solve the inner problems and hence determine the 
coefficients $b_{n}^{i}$ for $0 \leq n \leq i$. In practice, these coefficients are obtained by using Proposition 4 after the inner and the outer problems have been solved with a finite element method. The advantage is that those problems do not contain a small defect and the accuracy is guaranteed.

In Tabs. 4 and 5 are given the computed values of the first coefficients of the inner and outer expansions (with $H=1, L=5, \mu=1$ ). These coefficients are necessary to compute the first terms of the expansion of the energy and of the energy release rate.

Table 4. The computed values of the coefficients $b_{n}^{n}$ and $b_{n}^{n+2}$ for $0 \leq n \leq 4$ and of the leading terms $P^{0}$ and $P^{2}$ of the expansion of the potential energy for several values of the angle of the notch

\begin{tabular}{ccccccccccccc}
\hline$\epsilon$ & $b_{0}^{0}$ & $b_{1}^{1}$ & $b_{2}^{2}$ & $b_{3}^{3}$ & $b_{4}^{4}$ & $P^{0}$ & $b_{0}^{2}$ & $b_{1}^{3}$ & $b_{2}^{4}$ & $b_{3}^{5}$ & $b_{4}^{6}$ & $P^{2}$ \\
\hline 0 & 0 & -0.7834 & 0 & 0.6940 & 0 & 0.6940 & 0 & 0.2384 & 0 & 0.1058 & 0 & -0.4836 \\
0.1 & 0 & -0.7482 & 0 & 0.6606 & 0 & 0.6606 & 0 & 0.2091 & 0 & 0.0992 & 0 & -0.4413 \\
0.2 & 0 & -0.7089 & 0 & 0.6238 & 0 & 0.6238 & 0 & 0.1777 & 0 & 0.0905 & 0 & -0.3957 \\
0.3 & 0 & -0.6657 & 0 & 0.5847 & 0 & 0.5847 & 0 & 0.1451 & 0 & 0.0800 & 0 & -0.3486 \\
0.4 & 0 & -0.6187 & 0 & 0.5420 & 0 & 0.5420 & 0 & 0.1125 & 0 & 0.0683 & 0 & -0.3005 \\
\hline
\end{tabular}

Table 5. The computed values of the coefficients $a_{n}^{n+1}$ for $1 \leq n \leq 5$

\begin{tabular}{cccccc}
\hline$\epsilon$ & $a_{1}^{2}$ & $a_{2}^{3}$ & $a_{3}^{4}$ & $a_{4}^{5}$ & $a_{5}^{6}$ \\
\hline 0 & -0.3930 & 0 & 0.0987 & 0 & -0.0494 \\
0.1 & -0.3756 & 0 & 0.0943 & 0 & -0.0472 \\
0.2 & -0.3559 & 0 & 0.0893 & 0 & -0.0446 \\
0.3 & -0.3342 & 0 & 0.0838 & 0 & -0.0418 \\
0.4 & -0.3106 & 0 & 0.0778 & 0 & -0.0389 \\
\hline
\end{tabular}

The graphs of $\ell \mapsto \mathcal{P}^{\ell}$ and $\ell \mapsto \mathcal{G}^{\ell}$ obtained from these expansions are plotted on Fig. 5. They are compared with the values obtained directly by the finite element code Comsol, see [11]. It turns out that Comsol is not able to give accurate results for small values of $\ell$ (here when $\ell<0.01$ ) whereas the matching asymptotic expansion is valid in this range of values (and even the smaller $\ell$, the more accurate the asymptotic method). The two methods give the same results in the intermediate range of values $(0.01,0.1)$ of $\ell$. Note however that the asymptotic method diverges from the finite element method for large values of $\ell$. It is due to the fact that one has only computed the first terms of the expansion and one should compute more terms to obtain accurate results. Fig. 6 sketches the graph of $\mathcal{G}^{\ell}$ corresponding to the expansion of matching asymptotic up to different orders, specifically, up to the second, the fourth and the sixth term. Moreover, comparing with the result which is generated by FEM, see [11], one can see that the accuracy of the result increases with the number of terms included in the expansion. 

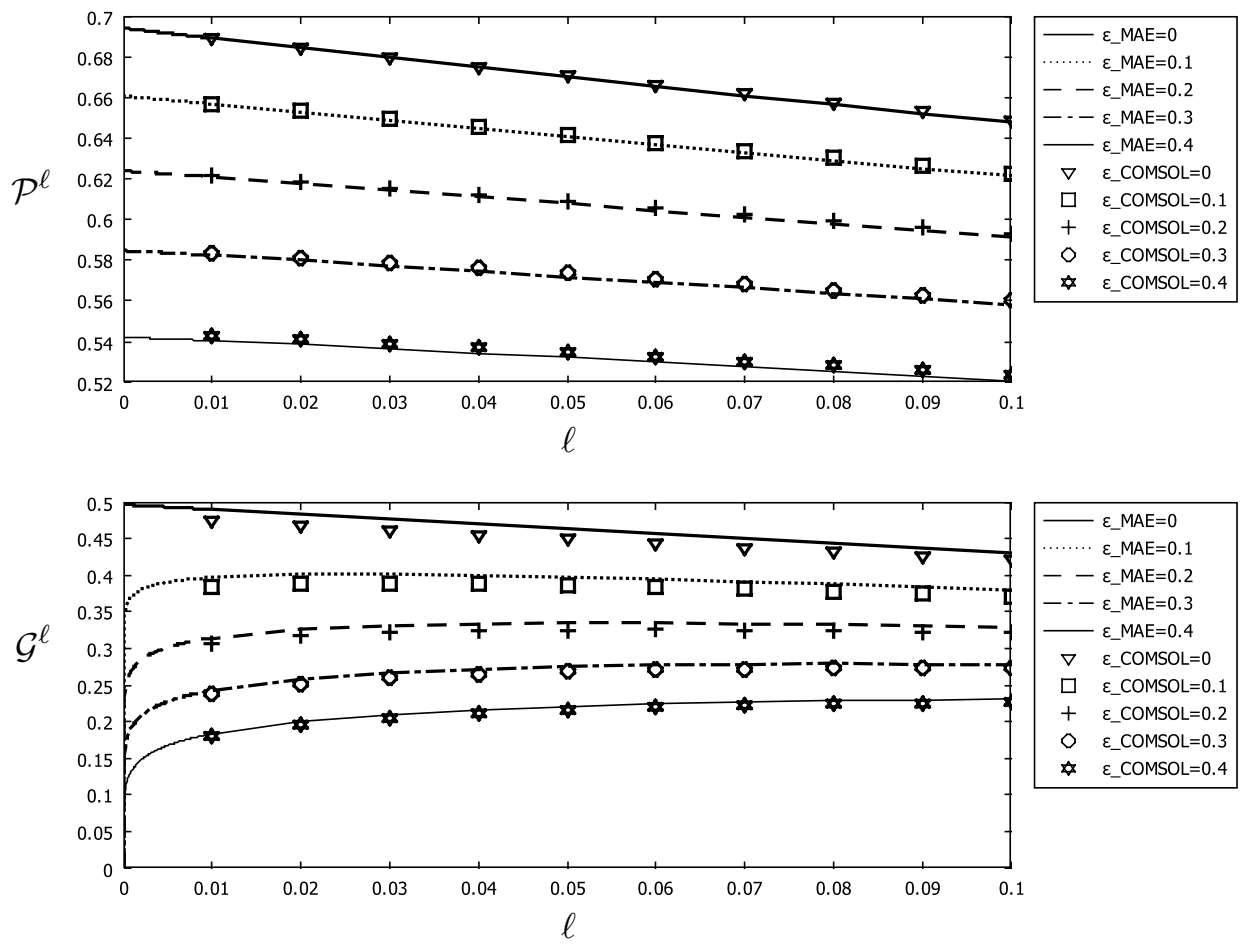

Fig. 5. Comparisons of the graphs of $\mathcal{P}^{\ell}$ and $\mathcal{G}^{\ell}$ near $\ell=0$ obtained by Matching Asymptotic Expansion or by the finite element code COMSOL for five values of the angle of the notch

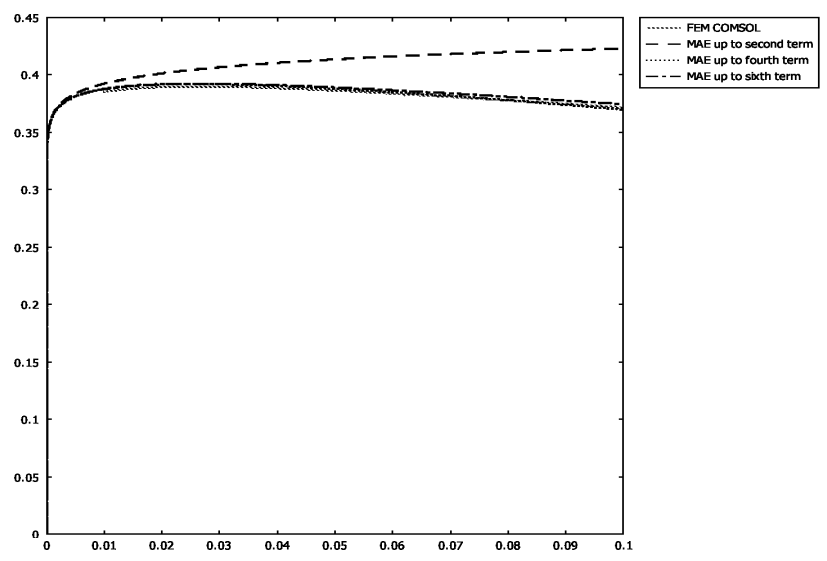

Fig. 6. The graphs of $\mathcal{G}^{\ell}$, when $\epsilon=0.1$, obtained by using either the Finite Element Method or the Asymptotic Method expanded up to the sixth order 


\section{CONCLUSION}

We have presented here a general method based on matched asymptotic expansions which can be applied to determine the mechanical fields and all related mechanical quantities in the case of a defect located at the tip of a notch. Applying this method to the case of a noncohesive crack, it turns out that it is sufficient to solve few inner and outer problems to obtain with very good accuracy the dependence of the energy and the energy release rate on the length of the crack. Moreover, this approximation can be used for very small values of the length of the crack and hence for determining the onset of the cracking whereas a classical finite element method gives rise to inaccurate results.

\section{REFERENCES}

[1] B. Bourdin, G. A. Francfort, and J.-J. Marigo. The variational approach to fracture. Journal of Elasticity, 91, (1-3), (2008), pp. 5-148. doi:10.1007/s10659-007-9107-3.

[2] A. Chambolle, A. Giacomini, and M. Ponsiglione. Crack initiation in brittle materials. Archive for Rational Mechanics and Analysis, 188, (2), (2008), pp. 309-349. doi:10.1007/s00205-007-00806.

[3] M. Charlotte, G. Francfort, J. J. Marigo, and L. Truskinovsky. Revisiting brittle fracture as an energy minimization problem: comparison of Griffith and Barenblatt surface energy models. Continuous Damage and Fracture, (2000), pp. 7-18.

[4] G. A. Francfort and J.-J. Marigo. Revisiting brittle fracture as an energy minimization problem. Journal of the Mechanics and Physics of Solids, 46, (8), (1998), pp. 1319-1342. doi:10.1016/s0022-5096(98)00034-9.

[5] H. D. Bui. Mécanique de la rupture fragile. Masson, Paris, (1978).

[6] B. Lawn. Fracture of brittle solids. Cambridge University Press, second edition, (1993).

[7] H. Ferdjani, R. Abdelmoula, and J.-J. Marigo. Insensitivity to small defects of the rupture of materials governed by the Dugdale model. Continuum Mechanics and Thermodynamics, 19, (3-4), (2007), pp. 191-210. doi:10.1007/s00161-007-0051-z.

[8] T. H. Pham, J. Laverne, and J.-J. Marigo. Stress gradient effects on the nucleation and propagation of cohesive cracks. Discrete and Continuous Dynamical Systems-Series S, 9, (2), (2016), pp. 557-584. doi:10.3934/dcdss.2016012.

[9] A. Chambolle, G. A. Francfort, and J.-J. Marigo. Revisiting energy release rates in brittle fracture. Journal of Nonlinear Science, 20, (4), (2010), pp. 395-424. doi:10.1007/s00332-010-90612.

[10] P. Destuynder, P. E. M. Djaoua, L. Chesnay, and J. C. Nedelec. Sur une interprétation mathématique de l'intégrale de rice en théorie de la rupture fragile. Mathematical Methods in the Applied Sciences, 3, (1), (1981), pp. 70-87. doi:10.1002/mma.1670030106.

[11] J.-J. Marigo. Initiation of cracks in Griffiths theory: an argument of continuity in favor of global minimization. Journal of Nonlinear Science, 20, (6), (2010), pp. 831-868. doi:10.1007/s00332-010-9074-x.

[12] P. Grisvard. Elliptic problems in nonsmooth domains. Pitman Publishing Inc., Marshfield, Massachusettes, (1985).

[13] P. Grisvard. Problemes aux limites dans les polygones; mode demploi. Bulletin de la Direction des Etudes et Recherches Series C, 1, (1986), pp. 21-59.

[14] P. Grisvard. Singularities in boundary value problems. Masson, (1992). 
[15] M. David, J.-J. Marigo, and C. Pideri. Homogenized interface model describing inhomogeneities located on a surface. Journal of Elasticity, 109, (2), (2012), pp. 153-187. doi:10.1007/s10659-012-9374-5.

[16] G. Geymonat, S. Hendili, F. Krasucki, and M. Vidrascu. Matched asymptotic expansion method for a homogenized interface model. Mathematical Models and Methods in Applied Sciences, 24, (3), (2014), pp. 573-597. doi:10.1142/s0218202513500607.

[17] G. Geymonat, F. Krasucki, S. Hendili, and M. Vidrascu. The matched asymptotic expansion for the computation of the effective behavior of an elastic structure with a thin layer of holes. International Journal for Multiscale Computational Engineering, 9, (5), (2011). doi:10.1615/intjmultcompeng.2011002619.

[18] J. K. Kevorkian and J. D. Cole. Multiple scale and singular perturbation methods, Vol. 114. Springer, (1996).

[19] D. Leguillon. Calcul du taux de restitution de l'énergie au voisinage d'une singularité. Comptes rendus de l'Académie des sciences. Série 2, 309, (10), (1989), pp. 945-950.

[20] J.-J. Marigo and C. Pideri. The effective behavior of elastic bodies containing microcracks or microholes localized on a surface. International Journal of Damage Mechanics, 20, (8), (2011), pp. 1151-1177. doi:10.1177/1056789511406914.

[21] M. Vidrascu, G. Geymonat, S. Hendili, and F. Krasucki. Matched asymptotic expansion and domain decomposition for an elastic structure. In 21st International Conference on Domain Decomposition Methods. Rennes, France, (2012).

[22] V. Bonnaillie-Noël, M. Dambrine, and G. Vial. Small defects in mechanics. In Internation Conference on Numerical Analysis and Applied Mathematics. AIP, (2011), pp. 1416-1419. doi:10.1063/1.3637887.

[23] M. Dauge. Elliptic boundary value problems on corner domains: smoothness and asymptotics of solutions. Springer Verlag, (1988).

[24] M. Dauge, S. Tordeux, and G. Vial. Selfsimilar perturbation near a corner: matching versus multiscale expansions for a model problem. In Around the research of Vladimir Maz'ya. II. Springer, (2010), pp. 95-134.

[25] H. Brezis. Functional analysis, Sobolev spaces and partial differential equations. Springer Science \& Business Media, (2010). doi:10.1007/978-0-387-70914-7.

[26] W. Schumann. Some basic problems of mathematical theory of elasticity. P. Noordhoff Ltd, Groningen, (1963).

[27] J. R. Rice. A path independent integral and the approximate analysis of strain concentration by notches and cracks. Journal of Applied Mechanics, 35, (2), (1968), pp. 379-386. doi:10.1115/1.3601206.

[28] A. A. Griffith and M. Eng. The phenomena of rupture and flow in solids. Philosophical Transactions of the Royal Society A, CCXXI, (1921), pp. 163-198. doi:10.1098/rsta.1921.0006.

[29] G. R. Irwin. Fracture. Handbuch der Physik, 6, (1958), pp. 551-590.

[30] Q. S. Nguyen. Stability and nonlinear solid mechanics. Wiley, (2000). 